Faisal Maqbool Zahid

\title{
Ordinal Ridge Regression with Categorical Predictors
}

Technical Report Number 101, 2011

Department of Statistics

University of Munich

http://www.stat.uni-muenchen.de 


\title{
Ordinal Ridge Regression with Categorical Predictors
}

\author{
Faisal Maqbool Zahida,*
}

${ }^{a}$ Ludwig-Maximilians-University Munich, Ludwigstrasse 33, D-80539 Munich, Germany.

\begin{abstract}
In multi-category response models categories are often ordered. In case of ordinal response models, the usual likelihood approach becomes unstable with ill-conditioned predictor space or when the number of parameters to be estimated is large relative to the sample size. The likelihood estimates do not exist when the number of observations is less than the number of parameters. The same problem arises if constraint on the order of intercept values is not met during the iterative procedure. Proportional odds models are most commonly used for ordinal responses. In this paper penalized likelihood with quadratic penalty is used to address these issues with a special focus on proportional odds models. To avoid large differences between two parameter values corresponding to the consecutive categories of an ordinal predictor, the differences between the parameters of two adjacent categories should be penalized. The considered penalized likelihood function penalizes the parameter estimates or differences between the parameters estimates according to the type of predictors. Mean squared error for parameter estimates, deviance of fitted probabilities and prediction error for ridge regression are compared with usual likelihood estimates in a simulation study and an application.
\end{abstract}

Key words: Likelihood estimation, Logistic regression, Non-proportional odds model, Partial proportional odds model, Penalization, Proportional odds model, Ridge regression.

\section{Introduction}

In regression analysis, maximum likelihood estimation is a common approach to compute the parameter estimates in categorical response models. But this approach fails if we have to estimate the parameters which are large in number relative to the sample size. In other words for a small ratio of sample size to the number of parameters (also for $p>n$ case) usual likelihood approach does not lead to a unique solution. The analyst faces the same problem for the data set with high correlation among the covariates and/or if there is a complete separation among the categories of the response variable. An alternative to the usual likelihood approach is to use penalized likelihood function. Penalization

${ }^{*}$ Corresponding author. Tel.: ++49 892180 6408; fax.:++49 8921805040.

Email addresses: faisal-maqbool.zahid@stat. uni-muenchen.de (Faisal Maqbool Zahid) 
techniques combine log-likelihood function with a penalty term which measures the smoothness of the fit. In recent years several penalization techniques with different types of penalties have been proposed. The main objective of using penalized log-likelihood is to obtain unique estimates of the parameters, better prediction with a good compromise between bias and variance, and/or to have a sparse model for clear and easy interpretation of the parameter estimates. Ridge regression is the most familiar penalization approach in the literature. In the context of linear models much literature is available for ridge regression. Schaefer et al. (1984) and Schaefer (1986) discussed the ridge penalty for logistic regression with binary response. An extension of ridge regression for GLM type models is considered by Nyquist (1991). LeCessie and Houwelingen (1992) discussed different ways to select the ridge penalty and also for computing prediction error in case of logistic ridge regression. In the literature univariate GLM's are more focused than the multivariate GLM. Zhu and Hastie (2004) used penalized logistic regression with quadratic penalty as an alternative to the support vector machine (SVM) for microarray cancer diagnostic problems. Zahid and Tutz (2009) used ridge penalty to get penalized estimates for logistic regression with multi-category (unordered) responses, which are independent of choice of the reference category. Ridge regression shrinks the parameter estimates to zero but none of them is exactly zero. As a result we do not have a parsimonious model but a model with all predictors. Another penalization approach called Lasso was proposed by Tibshirani (1996). Lasso technique not only shrinks the parameter estimates to zero but also serves as subset selection by setting some of the estimates exactly to zero. The lasso approach for multinomial logit models was considered by Friedman et al. (2010). In many applications multi-category responses are ordered. According to our knowledge penalization has not been addressed for ordered category response models. For ordinal responses there were several models discussed in the literature (see McCullagh (1980), Ananth and Kleinbaum (1997) and Agresti (1999)). However the proportional odds model (also known as cumulative logit models) is the most most popular among all other models for ordered category responses. Unlike multinomial logit models the proportional odds model (POM) has simple form in the sense that it has so-called global parameter estimates which are not category specific. But still in the case of large number of covariates maximum likelihood estimates may not exist. To resolve this problem we are using penalized log-likelihood with quadratic penalty to compute the estimates in proportional odds models. If the response variable $Y$ has $k$ ordered categories as $1, \ldots, k$, the general form of the cumulative logit model is given by

$$
\log \left[\frac{P(Y \leq r \mid \mathbf{x})}{P(Y>r \mid \mathbf{x})}\right]=\gamma_{0 r}-\mathbf{x}^{T} \boldsymbol{\gamma}_{r}, \quad r=1, \ldots, q=k-1 .
$$

The vector $\boldsymbol{\gamma}_{r}^{T}=\left(\gamma_{1 r}, \ldots, \gamma_{p r}\right)$ represents the category specific parameters. The simple form of proportional odds models with identical parameters for each category is given by

$$
\log \left[\frac{P(Y \leq r \mid \mathbf{x})}{P(Y>r \mid \mathbf{x})}\right]=\gamma_{0 r}-\mathbf{x}^{T} \boldsymbol{\gamma}, \quad r=1, \ldots, q=k-1 .
$$

Here the so-called global parameters vector $\boldsymbol{\gamma}^{T}=\left(\gamma_{1}, \ldots, \gamma_{p}\right)$ does not depend on the category. The ordered response $Y$ can be viewed as a categorized version of an unobservable latent variable $Z$ as $Y=r \Leftrightarrow \gamma_{0, r-1}<Z \leq \gamma_{0 r}$ for $r=$ $1, \ldots, k$, where $-\infty=\gamma_{00}<\gamma_{01}<\ldots<\gamma_{0 k}=\infty$ define the category boundaries on the unobservable latent 
continuum. The simple form of proportional odds model in (2) can be viewed as a univariate regression model for the latent variable with simple interpretation of parameters. The intercepts $\left\{\gamma_{0 r}\right\}$ are different for each cummulative logit and must satisfy the stochastic ordering $\gamma_{01}<\ldots<\gamma_{0 q}$ to have positive probabilities. Also the negative sign in (1) and (2) ensures that the probability is increasing for large values of $\mathbf{x}^{T} \boldsymbol{\gamma}$ with increasing $r$.

In the following text penalized estimates are computed using the penalized log-likelihood based on quadratic penalty for the simple form of the proportional odds models given in (2). In Section 2 penalized likelihood is discussed with some computational issues. The penalization with non-proportional odds models (NPOM) and partial proportional odds models (PPOM) is also described in this section. Empirical results of ordinal ridge regression are compared with usual ML estimates in Section 3. The ordinal ridge regression is fitted and compared with MLE using a real data in Section 4. The need of using penalized likelihood is also indicated in this section by drawing two small random samples of size $n=30$ and $n=50$ from the considered real data where usual MLE is not existing. Some final and concluding remarks are given in Section 5 .

\section{Penalization and Computational Issues}

For the proportional odds model given in (2), let $\phi_{i r}(\mathbf{x})$ denote the cumulative probability for the occurrence of response levels up to and including $r$ th level with a given covariate vector $\mathbf{x}_{i}$ given as

$$
\phi_{i r}(\mathbf{x})=P\left(Y_{i} \leq r \mid \mathbf{x}_{i}\right)=F\left(\eta_{i r}\right) \quad r=1, \ldots, q=k-1,
$$

where $F$ is a strictly monotone distribution function. The model with homogeneous effects has the predictor

$$
\eta_{i r}=\gamma_{0 r}+\mathbf{x}_{i}^{T} \boldsymbol{\gamma}
$$

In the context of multivariate generalized linear models, the simple proportional odds model can be given as

$$
\boldsymbol{\pi}_{i}=h\left(\mathbf{X}_{i} \boldsymbol{\beta}\right) \text { or } g\left(\boldsymbol{\pi}_{i}\right)=\mathbf{X}_{i} \boldsymbol{\beta},
$$

where $\boldsymbol{\pi}_{i}^{T}=\left(\pi_{i 1}, \ldots, \pi_{i q}\right)$ with components $\pi_{i r}=P\left(Y_{i}=r \mid \mathbf{x}_{i}\right)$ and $g=\left(g_{1}, \ldots, g_{k-1}\right)$ is a logit link type function given by

$$
g_{r}\left(\boldsymbol{\pi}_{i}\right)=\log \left[\log \left(\frac{\phi_{i r}}{1-\phi_{i r}}\right)-\log \left(\frac{\phi_{i, r-1}}{1-\phi_{i, r-1}}\right)\right] .
$$

For $p^{*}=p+q, \mathbf{X}_{i}=\left[\mathbf{I}_{q \times q}, \mathbf{1}_{q \times 1} \otimes \mathbf{x}_{i}^{T}\right]$ is a $q \times p^{*}$ matrix and $\boldsymbol{\beta}^{T}=\left(\boldsymbol{\gamma}_{0}^{T}, \boldsymbol{\gamma}^{T}\right)=\left(\gamma_{01}, \ldots, \gamma_{0 q}, \gamma_{1}, \ldots, \gamma_{p}\right)$ is a $p^{*} \times 1$ vector. The complete design matrix of order $n q \times p^{*}$ is given as $\mathbf{X}^{T}=\left[\mathbf{X}_{1}, \ldots, \mathbf{X}_{n}\right]$. For further details see McCullagh and Nelder (1989) and Fahrmeir and Tutz (2001).

The predictor space may contain some categorical predictors with more than one parameters associated with it. Let we have $K_{j}$ parameters associated with predictor $\mathbf{x}_{j}$. So for a binary or continuous covariate we have $K_{j}=1$ and 
if covariate is categorical then $K_{j}>1$ depending upon the number of categories of the predictor $\mathbf{x}_{j}$. The penalized $\log$-likelihood with quadratic penalty is given as

$$
l_{p}(\boldsymbol{\beta})=l(\boldsymbol{\beta})-\frac{\lambda}{2} J(\boldsymbol{\beta})
$$

where $l(\boldsymbol{\beta})$ is the usual log-likelihood function given by

$$
l(\boldsymbol{\beta})=\sum_{i=1}^{n} \sum_{r=1}^{k} y_{i r} \log \left(\pi_{i r}\right),
$$

and $\lambda$ is a tuning parameter. The penalty term $J(\boldsymbol{\beta})$ can be given as

$$
J(\boldsymbol{\beta})=\boldsymbol{\beta}^{T} \mathbf{P} \boldsymbol{\beta} .
$$

The definition of penalty matrix $\mathbf{P}$ depends on how we perform penalization with different types of predictors. If we have some ordinal predictors in the predictor space, penalization should be applied in such a way that ordinal covariates can be distinguished from the other covariates by considering the order of the categories. In penalization, it is common practice to penalize the parameter estimates but with ordinal predictors rather than estimates, difference between the parameters estimates of adjacent categories should be penalized (see Gertheiss and Tutz (2009)). Penalizing such differences will cause a smoothed version of the parameter estimates by avoiding the large difference among the estimates associated with the dummies of the ordinal predictors. Let the predictor $\mathbf{x}_{j}$ is ordinal with $K_{j}+1$ categories and first category is treated as reference category. If $\boldsymbol{\gamma}_{j}$ denotes the parameter vector for $K_{j}$ parameters/dummies associated with ordinal predictor $\mathbf{x}_{j}$, the penalty term for penalizing the differences between parameters of adjacent categories takes the form as

$$
J\left(\boldsymbol{\gamma}_{j}\right)=\sum_{l=2}^{K+1}\left(\gamma_{j l}-\gamma_{j-1, l}\right)^{2}=\boldsymbol{\gamma}_{j}^{T} \boldsymbol{\Omega}_{j} \boldsymbol{\gamma}_{j},
$$

with $\boldsymbol{\Omega}_{j}=\mathbf{U}_{j}^{T} \mathbf{U}_{j}$, for a $K_{j} \times K_{j}$ matrix $\mathbf{U}_{j}$ given by

$$
\mathbf{U}_{j}=\left[\begin{array}{ccccc}
1 & 0 & \cdots & \cdots & 0 \\
-1 & 1 & \ddots & & \vdots \\
0 & -1 & 1 & \ddots & \vdots \\
\vdots & \ddots & \ddots & \ddots & 0 \\
0 & \cdots & 0 & -1 & 1
\end{array}\right] .
$$

If the predictor is not ordinal then the penalty term for predictor $\mathbf{x}_{j}$ is given by

$$
J\left(\boldsymbol{\gamma}_{j}\right)=\sum_{l=2}^{K+1} \gamma_{j l}^{2}=\boldsymbol{\gamma}_{j}^{T} \mathbf{I}_{j} \boldsymbol{\gamma}_{j},
$$

where $\mathbf{I}_{j}$ is a $K_{j} \times K_{j}$ identity matrix. For the predictor space with all types of predictors (i.e., binary/continuous, nominal and ordinal predictors), the penalty matrix $\mathbf{P}$ given in (7) is given by

$$
\mathbf{P}=\underset{4}{\operatorname{diag}\left(\mathbf{0}_{q \times q}, \mathbf{P}_{1}, \ldots, \mathbf{P}_{p}\right)}
$$


Here $\mathbf{0}_{q \times q}$ is a zero matrix with zeros for the category specific intercept terms which are not penalized. If the $j$ th predictor is ordinal then $\left(K_{j} \times K_{j}\right)$-submatrix $\mathbf{P}_{j}$ assumes the value $\boldsymbol{\Omega}_{j}$ otherwise $\mathbf{P}_{j}=\mathbf{I}_{j}$.

The penalized log-likelihood function given in (5) can be written as

$$
l_{p}(\boldsymbol{\beta})=\sum_{i=1}^{n} l_{i}(\boldsymbol{\beta})-\frac{\lambda}{2} \sum_{j=1}^{p} \boldsymbol{\beta}_{j}^{T} \mathbf{P} \boldsymbol{\beta}_{j}
$$

Score function $s_{p}(\boldsymbol{\beta})$ for the penalized log-likelihood is given by

$$
\begin{aligned}
s_{p}(\boldsymbol{\beta}) & =\sum_{i=1}^{n} \mathbf{X}_{i}^{T} \mathbf{D}_{i}(\boldsymbol{\beta}) \boldsymbol{\Sigma}_{i}^{-1}(\boldsymbol{\beta})\left[\mathbf{y}_{i}-h\left(\boldsymbol{\eta}_{i}\right)\right]-\lambda \mathbf{P} \boldsymbol{\beta} \\
& =\mathbf{X}^{T} \mathbf{D}(\boldsymbol{\beta}) \boldsymbol{\Sigma}^{-1}(\boldsymbol{\beta})[\mathbf{y}-h(\boldsymbol{\eta})]-\lambda \mathbf{P} \boldsymbol{\beta}
\end{aligned}
$$

where $\mathbf{D}_{i}(\boldsymbol{\beta})=\frac{\partial h\left(\boldsymbol{\eta}_{i}\right)}{\partial \boldsymbol{\eta}}$ is derivative of $h(\boldsymbol{\eta})$ evaluated at $\boldsymbol{\eta}_{i}=\mathbf{X}_{i} \boldsymbol{\beta}, \boldsymbol{\Sigma}_{i}(\boldsymbol{\beta})=\operatorname{cov}\left(\mathbf{y}_{i}\right)$ is the covariance matrix of $i$ th observation of $\mathbf{y}$ given parameter vector $\boldsymbol{\beta}$ and $\mathbf{W}_{i}(\boldsymbol{\beta})=\mathbf{D}_{i}(\boldsymbol{\beta}) \boldsymbol{\Sigma}_{i}^{-1} \mathbf{D}_{i}^{T}(\boldsymbol{\beta})$. $\mathbf{y}$ and $h(\boldsymbol{\eta})$ are given by $\mathbf{y}^{T}=\left(\mathbf{y}_{1}^{T}, \ldots, \mathbf{y}_{n}^{T}\right)$ and $h(\boldsymbol{\eta})^{T}=\left(h\left(\boldsymbol{\eta}_{1}\right)^{T}, \ldots, h\left(\boldsymbol{\eta}_{n}\right)^{T}\right)$. The matrices have block diagonal form $\boldsymbol{\Sigma}(\boldsymbol{\beta})=\operatorname{diag}\left(\boldsymbol{\Sigma}_{i}^{-1}(\boldsymbol{\beta})\right), \mathbf{D}(\boldsymbol{\beta})=\operatorname{diag}\left(\mathbf{D}_{i}(\boldsymbol{\beta})\right)$ and $\mathbf{W}(\boldsymbol{\beta})=\operatorname{diag}\left(W_{i}(\boldsymbol{\beta})\right)$. By equating the score function to zero we obtain the estimation equations as

$$
\mathbf{X}^{T} \mathbf{D}(\boldsymbol{\beta}) \boldsymbol{\Sigma}^{-1}(\boldsymbol{\beta})[\mathbf{y}-h(\boldsymbol{\eta})]-\lambda \mathbf{P} \boldsymbol{\beta}=\mathbf{0},
$$

where $\boldsymbol{\beta}$ is $p^{*} \times 1$ parameter vector for $p^{*}=q+\sum_{j=1}^{p} K_{j}$ and $\mathbf{P}$ is a $p^{*} \times p^{*}$ matrix given in (8). Fisher scoring iteration yields

$$
\hat{\boldsymbol{\beta}}^{(k+1)}=\hat{\boldsymbol{\beta}}^{(k)}+\left(\mathbf{X}^{T} \mathbf{W}\left(\hat{\boldsymbol{\beta}}^{(k)}\right) \mathbf{X}+\lambda \mathbf{P}\right)^{-1} s_{p}\left(\hat{\boldsymbol{\beta}}^{(k)}\right) .
$$

If $\hat{\boldsymbol{\beta}}$ are penalized estimates for the true parameter $\boldsymbol{\beta}$, the covariance matrix can be approximated by

$$
\operatorname{cov}(\hat{\boldsymbol{\beta}})=\left(\mathbf{X}^{T} \mathbf{W}(\hat{\boldsymbol{\beta}}) \mathbf{X}+\lambda \mathbf{P}\right)^{-1}\left(\mathbf{X}^{T} \mathbf{W}(\hat{\boldsymbol{\beta}}) \mathbf{X}\right)\left(\mathbf{X}^{T} \mathbf{W}(\hat{\boldsymbol{\beta}}) \mathbf{X}+\lambda \mathbf{P}\right)^{-1} .
$$

Generalized cross-validation (GCV) criterion is used to find the optimal value of ridge penalty $\lambda$. In the generalized linear models (GLM) environment we are using likelihood-based criterion deviance for GCV instead of squared distances of $y_{i}$ and $\pi_{i}$. Deviance based generalized cross-validation is given by

$$
\mathrm{GCV}=\frac{-2 \cdot \sum_{i=1}^{n}\left(l_{\lambda}\left(\hat{\boldsymbol{\pi}}_{i}\right)-l_{i}\left(\mathbf{y}_{i}\right)\right)}{(1-\operatorname{tr}(\mathbf{H}(\lambda)) / n)^{2}}
$$

where the the hat matrix $\mathbf{H}$ is given as

$$
\mathbf{H}=\mathbf{W}^{T / 2} \mathbf{X}\left(\mathbf{X}^{T} \mathbf{W}(\hat{\boldsymbol{\beta}}) \mathbf{X}+\lambda \mathbf{P}\right)^{-1} \mathbf{X}^{T} \mathbf{W}^{1 / 2} .
$$

In this text we are using penalization to address the problems with likelihood estimation for proportional odds models which are commonly used with ordered response categories. But some problems may become more critical when we are dealing with non-proportional odds models (NPOM) given in (1) with category specific parameters or partial proportional odds model (PPOM) given by

$$
\eta_{i r}=\gamma_{0 r}+\mathbf{x}_{i G}^{T} \boldsymbol{\gamma}_{G}+\mathbf{x}_{5}^{T} \boldsymbol{\gamma}_{L r} \quad r=1, \ldots, q,
$$


where some of the parameters have global effect $\gamma_{G}$ and others have local/category specific effect $\gamma_{L r}$. In both of these types of models we have to estimate more parameters than in the corresponding proportional odds model. In case of non-proportional or partial proportional odds models, penalization can be implemented in the same way as for POM. The only difference is that the structure of design matrix will be changed according to the model. In case of proportional odds model the design matrix for $i$ th observation is a $q \times p^{*}$ matrix $\mathbf{X}_{i}=\left[\mathbf{I}_{q \times q}, \mathbf{1}_{q \times 1} \otimes \mathbf{x}_{i}^{T}\right]$ with $p^{*}=p+q$, as discussed above. For non-proportional odds model the design matrix assumes the same structure as for multinomial logit models and for the $i$ th observation is given as $\mathbf{X}_{i}=\left[\mathbf{I}_{q \times q}, \mathbf{I}_{q \times q} \otimes \mathbf{x}_{i}^{T}\right]$. For partial proportional odds models the design matrix can be written as $\mathbf{X}_{i}=\left[\mathbf{I}_{q \times q}, \mathbf{1}_{q \times 1} \otimes \mathbf{x}_{G i}^{T}, \mathbf{I}_{q \times q} \otimes \mathbf{x}_{L i}^{T}\right]$ where $x_{G i}^{T}$ is a vector associated with predictors having global effect and $x_{L i}^{T}$ is a vector of observations for those predictors having local i.e., category specific effect. The parameter vector associated with POM, NPOM and PPOM are given by $\boldsymbol{\beta}^{T}=\left(\boldsymbol{\gamma}_{0}^{T}, \boldsymbol{\gamma}^{T}\right)=\left(\gamma_{01}, \ldots, \gamma_{0 q}, \gamma_{1}, \ldots, \gamma_{p}\right)$, $\boldsymbol{\beta}^{T}=\left(\boldsymbol{\gamma}_{0}^{T}, \boldsymbol{\gamma}_{1}^{T}, \ldots, \boldsymbol{\gamma}_{q}^{T}\right)$ and $\boldsymbol{\beta}^{T}=\left(\boldsymbol{\gamma}_{0}^{T}, \boldsymbol{\gamma}_{G}^{T}, \boldsymbol{\gamma}_{L 1}^{T}, \ldots, \boldsymbol{\gamma}_{L q}^{T}\right)$ respectively. In case of NPOM and PPOM, the penalty matrix $\mathbf{P}$ has a higher dimension than the proportional odds models and can be formulated according to the structure of design matrix based on the desired model to fit.

\section{Simulation Study}

The effectiveness of ridge regression is discussed in this section using simulated data. For a sample of size $n$ predictor space contains continuous covariates (denoted by $C$ ), binary covariates (denoted by $B$ ), nominal covariates (denoted by $N_{K+1}$ ) and/or ordinal covariates (denoted by $O_{K+1}$ ) with $K+1$ categories. The continuous covariates are drawn from a p-dimensional centered multivariate normal distribution with covariance between two covariates $\mathbf{x}_{j}$ and $\mathbf{x}_{k}$ being $\rho^{|j-k|}$. Four values of $\rho=0.0,0.3,0.7$ and 0.9 are used. To study the problems with existence of usual MLE, we consider proportional odds models with $k=3$ and $k=5$ response categories for each setting of predictor space given in Table 1. The true values used for the intercept terms are $(-0.3,0.8)$ and $(-0.8,-0.3,0.3,0.8)$ for $k=3$ and $k=5$ respectively. The true values for global parameters are obtained as $(-1)^{j} \exp (-2(j-1) / 20)$ for $j=1, \ldots, \sum_{j=1}^{p} K_{j}$. A multiplicative factor $c_{\mathrm{snr}}$ for $\boldsymbol{\beta}^{T}=\left(\boldsymbol{\gamma}_{0}^{T}, \boldsymbol{\gamma}\right)$ is chosen so that the signal-to-noise ratio is 1 . 


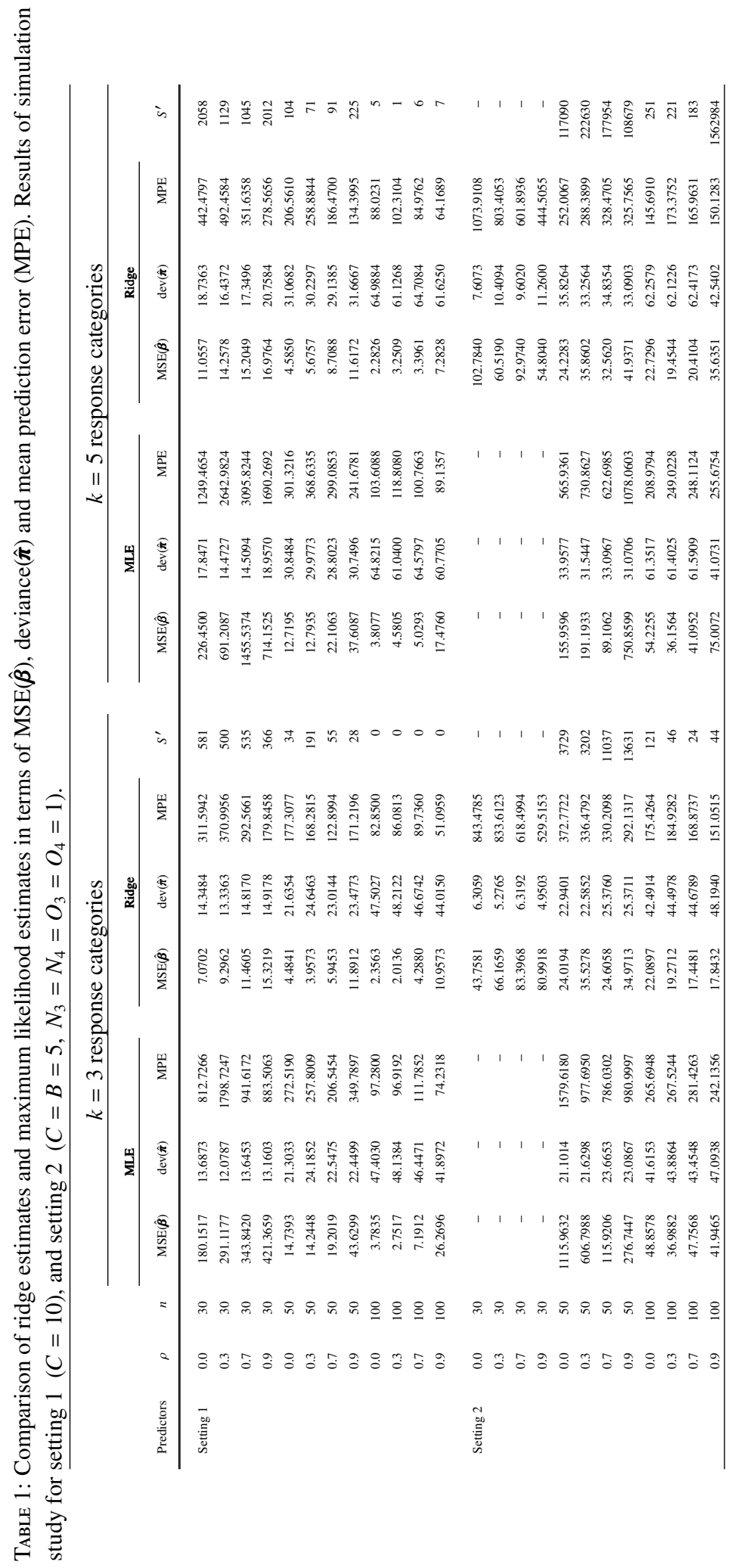


TABLE 2: Comparison of Bias in ridge estimates and maximum likelihood estimates. Results of simulation study for setting $1(C=10)$, and setting $2\left(C=B=5, N_{3}=N_{4}=O_{3}=O_{4}=1\right)$.

\begin{tabular}{|c|c|c|c|c|c|c|}
\hline \multirow[b]{2}{*}{ Predictors } & \multirow[b]{2}{*}{$\rho$} & \multirow[b]{2}{*}{$n$} & \multicolumn{2}{|c|}{$k=3$ response categories } & \multicolumn{2}{|c|}{$k=5$ response categories } \\
\hline & & & Bias (MLE) & Bias (Ridge) & Bias (MLE) & Bias (Ridge) \\
\hline \multirow[t]{12}{*}{ Setting 1} & 0.0 & 30 & 6.1013 & 2.4959 & 7.9885 & 2.9878 \\
\hline & 0.3 & 30 & 10.7727 & 2.8926 & 16.8054 & 3.5454 \\
\hline & 0.7 & 30 & 9.7730 & 3.2490 & 24.5497 & 3.7247 \\
\hline & 0.9 & 30 & 13.3824 & 3.8163 & 16.3382 & 3.9171 \\
\hline & 0.0 & 50 & 3.1968 & 2.0083 & 2.8157 & 1.9978 \\
\hline & 0.3 & 50 & 3.0562 & 1.9167 & 3.2015 & 2.2838 \\
\hline & 0.7 & 50 & 3.8132 & 2.3512 & 4.2058 & 2.8365 \\
\hline & 0.9 & 50 & 5.9668 & 3.2345 & 5.5624 & 3.3067 \\
\hline & 0.0 & 100 & 1.7180 & 1.4164 & 1.8226 & 1.4481 \\
\hline & 0.3 & 100 & 1.5501 & 1.3569 & 1.9473 & 1.6707 \\
\hline & 0.7 & 100 & 2.4421 & 1.9737 & 2.0823 & 1.7594 \\
\hline & 0.9 & 100 & 4.4083 & 3.1984 & 3.8908 & 2.6397 \\
\hline \multirow[t]{12}{*}{ Setting 2} & 0.0 & 30 & - & 6.4481 & - & 9.4966 \\
\hline & 0.3 & 30 & - & 7.9981 & - & 7.5097 \\
\hline & 0.7 & 30 & - & 8.9882 & - & 9.1005 \\
\hline & 0.9 & 30 & - & 8.7376 & - & 7.0519 \\
\hline & 0.0 & 50 & 28.9515 & 4.7621 & 10.8452 & 4.7540 \\
\hline & 0.3 & 50 & 16.5924 & 5.6959 & 12.2986 & 5.7770 \\
\hline & 0.7 & 50 & 9.2153 & 4.7695 & 8.7403 & 5.4500 \\
\hline & 0.9 & 50 & 13.9843 & 5.6826 & 20.4349 & 6.2991 \\
\hline & 0.0 & 100 & 6.5044 & 4.4605 & 6.6477 & 4.4492 \\
\hline & 0.3 & 100 & 5.8020 & 4.2456 & 5.6645 & 4.2098 \\
\hline & 0.7 & 100 & 6.2549 & 3.9546 & 5.9056 & 4.2600 \\
\hline & 0.9 & 100 & 6.1046 & 4.0720 & 7.9145 & 5.7814 \\
\hline
\end{tabular}

For each setting mentioned in Table 1 with different number and type of covariates, $S=50$ samples of size $n$ are used in the study. To compare the results of ridge estimates with likelihood estimates we consider only those samples for which ML estimates exist. In order to obtain $S=50$ samples, $S^{\prime}$ samples are ignored because ML estimates with their standard errors are not existing for these samples using polr function of library MASS in statistical language R 2.10.0. In Table 1, the columns with title $S^{\prime}$ showing the number of samples for which ML estimates did not exist, highlights the need of a penalization technique. The results of Table 1 show that value of $S^{\prime}$ is increasing with increasing number of predictors drawn from different distributions. This problem becomes even more severe with $k=5$ response categories. For sample size $n=30$, we cannot generate 50 samples for which MLE is existing in setting 2 and in setting 1 although estimates are presented but they have quite large standard errors (not shown here). Although ML estimates become more stable for independent predictors or in case of moderate correlation among predictors with increasing sample size, however they are deteriorated in case of high multicollinearity. The ridge regression may be the best choice to obtain stable estimates of parameters for all predictors in the model when usual MLE is not existing or deteriorated because of ill-conditioned predictor space. In Table 1, ML estimates are computed using polr function of statistical environment/language R 2.10.0. Ridge estimates are compared with likelihood estimates in terms of 

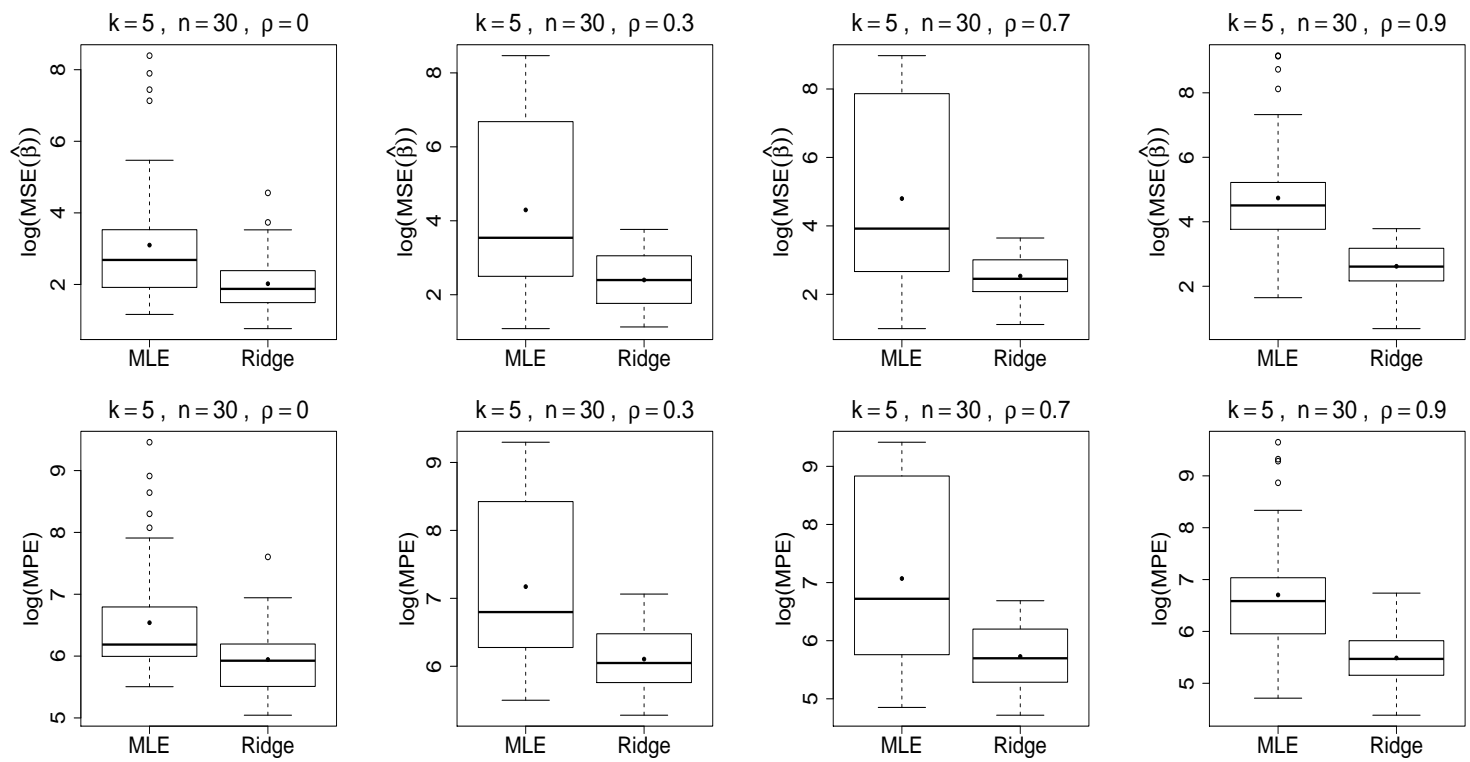

FIGURE 1: Illustration of simulation study for ten predictors drawn from multivariate normal distribution for $k=5$ response categories: Box plots for comparing ridge estimates with ML estimates in terms of $\log (\operatorname{MSE}(\hat{\boldsymbol{\beta}}))$ (top panel) and log values of Mean Prediction Error i.e., $\log$ (MPE) (bottom panel). The solid circles within the boxes represent the mean of the observations for which box plots are drawn.

mean squared error (MSE) of $\hat{\boldsymbol{\beta}}$, deviance of the fitted probabilities and mean prediction error (MPE). In all settings we use samples of size $n$ for the training data and then a sample of size $n_{\text {test }}=1000$ is generated for comparing the prediction performance of ridge estimates with likelihood estimates. The results of Table 1 show that ridge is performing better than MLE as is expected. In case of ten normally distributed covariates, ML estimates have very large standard errors for $n=30$. In the simulation setting 2 considered with categorical predictors there are twenty parameters to be estimated (other than intercept terms). Here the situation goes more worse with MLE and we have to leave a large number of samples (especially with $k=5$ response categories) because of problems with existence of estimates. Even we could not get enough samples with $n=30$ for which likelihood estimates with their standard errors exist. So for setting 2 with $n=30$ only the results of ridge regression are given in Table 1 . The ridge estimates do exist in all considered situations even when the likelihood estimates do not exist. For comparing ridge estimates with ML estimates, mean squared error is computed as $\frac{1}{S} \sum_{s}\left(\hat{\boldsymbol{\beta}}_{s}^{\text {method }}-\boldsymbol{\beta}^{\text {true }}\right)^{T}\left(\hat{\boldsymbol{\beta}}_{s}^{\text {method }}-\boldsymbol{\beta}^{\text {true }}\right)$ and the formula used to compute deviance of fit i.e., $\operatorname{Dev}(\hat{\boldsymbol{\pi}})$ is given by $D=2$. $\sum_{i=1}^{n} \sum_{j=1}^{k} y_{i j} \log \left(\frac{y_{i j}}{\hat{\pi}_{i j}}\right)$ with $y_{i j} \log \left(\frac{y_{i j}}{\hat{\pi}_{i j}}\right)=0$ for $y_{i j}=0$. Mean prediction error based on 1000 test observations is computed as MPE $=\frac{1}{S} \sum_{s} D_{s}=\frac{1}{S} \sum_{s} 2 \cdot\left[\sum_{i=1}^{n} \sum_{j=1}^{k} \pi_{i j s}^{\text {test }} \log \left(\frac{\pi_{i j s}^{\text {test }}}{\hat{\tilde{T}}_{i j s}^{\text {test }}}\right)\right]$. In addition to $\operatorname{MSE}(\hat{\boldsymbol{\beta}})$ it is also important to observe the amount of bias in the parameter estimates. The mean length of vectors of bias in $S=50$ samples is computed for ridge and ML estimates as $\frac{1}{S} \sum_{s}\left\|\hat{\boldsymbol{\beta}}_{s}^{\text {method }}-\boldsymbol{\beta}^{\text {true }}\right\|$. The mean values for lengths of bias vectors for MLE and ridge estimates is given in Table 2 for each setting of simulation study. The graphical representation of the results for more interesting cases with small samples for $k=5$ response categories 

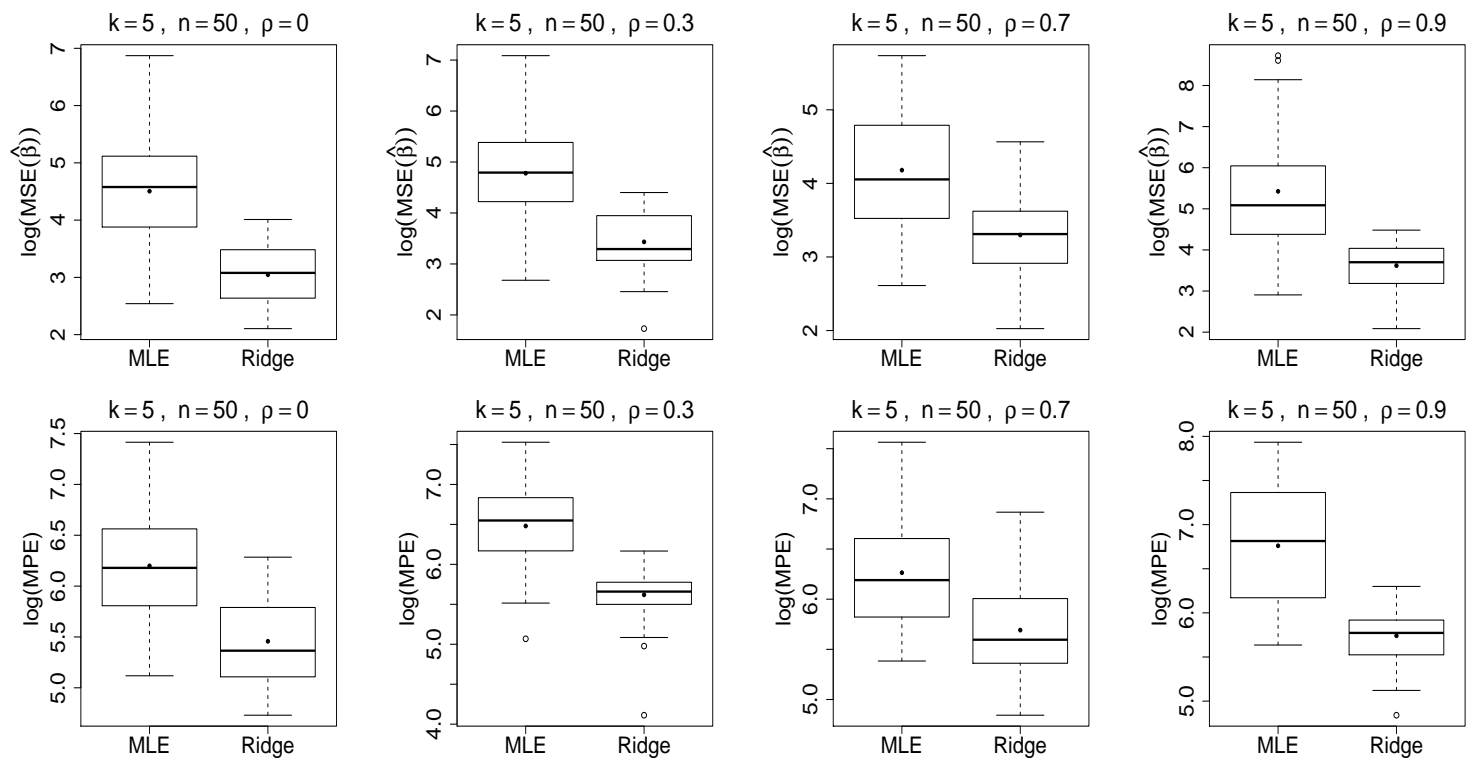

FIGURE 2: Illustration of simulation study for $k=5$ response categories and $C=B=5$ and $N_{3}=N_{4}=O_{3}=O_{4}=1$ predictors in the predictor space: Box plots for comparing ridge regression estimates with MLE in terms of $\log (\operatorname{MSE}(\hat{\boldsymbol{\beta}}))$ (top panel) and Mean Prediction Error i.e., $\log (\mathrm{MPE})$ (bottom panel). The solid circles within the boxes represent the mean of the observations for which box plots are drawn.

is given in Figure 1 and 2 with the help of box plots. The solid circles in each box represents the mean of the values for which box plot is drawn.

\section{Application}

In this section for computing and comparing the ridge estimates with ML estimates, we are considering the housing data set from UCI repository (http://archive.ics.uci.edu/ml/datasets/Housing). The response variable (MEDV) is about the median values of the owner-occupied houses in suburbs of Boston. For the analysis purpose, response variable which is measured in $\$ 1000$ 's is divided into four price categories as lower, lower middle, upper middle and high price category using the thresholds as MEDV $<10,10 \leq$ MEDV $<25,25 \leq$ MEDV $<40$ and MEDV $\geq 40$. The predictors used to predict the price range of a house are: per capita crime rate by town (CRIM); proportion of residential land zoned for lots over 25,000 sq.ft. (ZN); proportion of non-retail business acres per town (INDUS); Charles River dummy variable (CHAS = 1 if tract bounds river; CHAS = 0 otherwise); nitric oxides concentration (NOX)(parts per 10 million); average number of rooms per dwelling (RM); proportion of owner-occupied units built prior to 1940 (AGE); weighted distances to five Boston employment centres (DIS); index of accessibility to radial highways (RAD); full-value property-tax rate per $\$ 10,000$ (TAX); pupil-teacher ratio by town (PTRATIO); $1000(\mathrm{Bk}-0.63)^{2}$ where $\mathrm{Bk}$ is the proportion of blacks by town (B) and \% lower status of the population (LSTAT). 
Although the polr function of R provides the likelihood estimates of parameters but fails to produce standard errors of these estimates. So the usual ML estimates and corresponding standard errors are not computed with polr as in simulation study but ML estimates are computed for $\lambda=0$. For computing the ridge estimates, ridge penalty is decided on the basis of deviance based generalized cross-validation (GCV). The parameter estimates with their standard errors are given in Table 3 for the complete data set. The results of ridge regression are based on the optimal value of ridge penalty $\lambda=0.1$. In order to check the existence of MLE in case of small samples, different random samples of size $n=30$ are drawn from the complete data set and likelihood estimates are not existing for any of the these samples. Similarly ML estimates are not existing for most of the random samples of size $n=50$ drawn from total sample of size $n=506$. However ridge estimates are existing for all such small samples. Two such small samples with $n=30$ and $n=50$ are considered for which MLE is not existing but ridge estimates do exist. Both of these data sets can be accessed at www.stat.uni-muenchen.de/ zahid/samples_ordinalRidge.txt. The parameter estimates and their standard errors for ridge regression with these two samples are given in Table 3. The ridge estimates in both of these small samples are computed with optimal value of ridge penalty $\lambda=0.1$.

TABLE 3: MLE and ridge estimates with corresponding standard errors for complete housing data set $(n=506)$ and ridge estimates with their standard errors for random samples of size $n=30$ and $n=50$ drawn from the complete data set.

\begin{tabular}{|c|c|c|c|c|c|c|c|c|}
\hline & \multicolumn{2}{|c|}{$\operatorname{MLE}(n=506)$} & \multicolumn{2}{|c|}{ Ridge $(n=506)$} & \multicolumn{2}{|c|}{ Ridge $(n=30)$} & \multicolumn{2}{|c|}{ Ridge $(n=50)$} \\
\hline & Estimate & S.E. & Estimate & S.E. & Estimate & S.E. & Estimate & S.E. \\
\hline CRIM & 0.0816 & 0.0210 & 0.0804 & 0.0209 & 0.6898 & 0.9303 & -0.4804 & 0.4044 \\
\hline $\mathrm{ZN}$ & -0.0090 & 0.0075 & -0.0093 & 0.0075 & -1.4414 & 0.9211 & -0.0717 & 0.0498 \\
\hline INDUS & -0.0344 & 0.0393 & -0.0253 & 0.0384 & 0.6731 & 0.8954 & -0.7055 & 0.2939 \\
\hline CHAS & -0.7756 & 0.5078 & -0.7710 & 0.4909 & $-9.6 \mathrm{E}-10$ & 0.0001 & -2.2409 & 1.5557 \\
\hline NOX & 5.6532 & 2.6104 & 3.3769 & 1.5477 & 0.0113 & 0.0497 & 1.9551 & 0.8191 \\
\hline $\mathrm{RM}$ & -1.4677 & 0.2715 & -1.4615 & 0.2678 & -0.4699 & 0.2782 & -3.5199 & 1.3463 \\
\hline AGE & -0.0028 & 0.0077 & -0.0010 & 0.0075 & -0.5734 & 0.4378 & -0.0387 & 0.0379 \\
\hline DIS & 0.4321 & 0.1243 & 0.3970 & 0.1186 & -1.1194 & 1.1935 & -0.6395 & 0.5703 \\
\hline RAD & -0.2029 & 0.0475 & -0.1952 & 0.0465 & -0.3114 & 0.5659 & -0.5855 & 0.2925 \\
\hline TAX & 0.0077 & 0.0025 & 0.0077 & 0.0025 & 0.0394 & 0.0752 & 0.0386 & 0.0172 \\
\hline PTRATIO & 0.3756 & 0.0844 & 0.3501 & 0.0804 & 1.9405 & 1.2744 & 1.1177 & 0.4431 \\
\hline B & -0.0077 & 0.0022 & -0.0077 & 0.0022 & -0.2529 & 0.5010 & 0.0092 & 0.0102 \\
\hline LSTAT & 0.3082 & 0.0432 & 0.3119 & 0.0430 & 0.1750 & 0.8308 & 0.9086 & 0.3917 \\
\hline
\end{tabular}

\section{Concluding Remarks}

Ridge regression provides stable estimates in logistic regression when maximum likelihood estimates are deteriorated because of ill-conditioned predictor space. Multicollinearity among the predictors causes an increase in the average length of likelihood estimates of parameter vector and inflates the standard errors of these estimates. Also when 
sample size is small relative to the number of parameters, existing softwares for fitting proportional odds models may face the problems in computing the estimates and especially with computing standard errors of the estimates (e.g., polr function of MASS package in $\mathrm{R}$ that we used in this text may provide the parameter estimates for some data sets but fails to produce the corresponding standard errors). If $p^{*}>n$ the maximum likelihood estimates will not exist at all. If stochastic ordering of estimates for the intercepts is disturbed during iterative process, it will lead to illogical values of the fitted probabilities. To address all of these issues, ridge regression is used in this paper for ordinal response models with a focus on proportional odds models. However for non-proportional odds models or partial proportional odds models the ridge regression can be used easily in the same way. The only difference is that we have to penalize more parameter estimates depending upon the model/design space under consideration as discussed in the end of Section 2. Since ridge penalty shrinks the parameters estimates to zero but does not perform variable selection, it is useful in case of limited number of predictors where the analysts are interested in fitting a model by keeping all the predictors in the model. With penalization, parameter estimates corresponding to the categorical predictors are penalized by considering whether it is a nominal or ordinal predictor. For ordinal predictors differences between the parameters estimates of successive categories should be small. In this paper, these differences are kept small by penalizing the differences between successive parameters estimates of ordinal predictors instead of penalizing the estimates themselves.

\section{References}

Agresti, A., 1999. Modelling ordered categorical data: Recent advances and future challenges. Statistics in Medicine 18, $2191-2207$.

Ananth, C. V., Kleinbaum, D. G., 1997. Regression models for ordinal responses: A review of methods and applications. International Journal of Epidemiology 26, Number 6, 1323-1333.

Fahrmeir, L., Tutz, G., 2001. Multivariate Statistical Modelling based on Generalized Linear Models. second ed. Springer-Verlag NewYork, Inc.

Friedman, J., Hastie, T., Tibshirani, R., 2010. Regularization paths for generalized linear models via coordinate descent. Jounal of Statistical Software 33, Issue 1.

Gertheiss, J., Tutz, G., 2009. Penalized regression with ordinal predictors. International Statistical Review 77, 345-365.

LeCessie, S., Houwelingen, V., 1992. Ridge estimators in logistic regression. Applied Statistics 41, 191-201.

McCullagh, P., 1980. Regression models for ordinal data. Journal of the Royal Statistical Society B 42, 109-142.

McCullagh, P., Nelder, J., 1989. Generalized Linear Models. second ed. Chapman \& Hall, New York.

Nyquist, H., 1991. Restricted estimation of generalized linear models. Journal of Applied Statistics 40, $133-141$.

Schaefer, R., 1986. Alternative estimators in logistic regression when the data are collinear. Journal of Statistical Computation and Simulation 25, 75-91.

Schaefer, R., Roi, L., Wolfe, R., 1984. A ridge logistic estimator. Communications in Statistics: Theory and Methods 13, 99-113. 
Tibshirani, R., 1996. Regression shrinkage and selection via lasso. Journal of the Royal Statistical Society B 58, $267-288$.

Zahid, F. M., Tutz, G., 2009. Ridge estimation for multinomial logit models with symmetric side constraints. Technical Report No. 67. Institute of Statistics, Ludwig-Maximilians-University Munich, Germany.

Zhu, J., Hastie, T., 2004. Classification of gene microarrays by penalized logistic regression. Biostatistics 5, 427-443. 